\title{
Multiplicative Iteration On Infinite Products
}

Benedict Irwin ${ }^{1}$

${ }^{1}$ University of Cambridge

January 7, 2021

We have the product

$$
\prod_{n=2}^{\infty} \frac{n^{3}-1}{n^{3}+1}=\frac{2}{3}
$$

if we take the prodegrand and apply a multiplicative derivative defined by

$$
f^{*}(x)=\exp \left(\frac{f^{\prime}(x)}{f(x)}\right)
$$

then we get

$$
\prod_{n=2}^{\infty} \exp \left(\frac{6 n^{2}}{n^{6}-1}\right) \sim 1.64872
$$

This converges to

$$
\lim _{m \rightarrow \infty} \exp \left(\frac{m^{4}+2 m^{3}+2 m^{2}-3 m-2}{2 m(m+1)\left(m^{2}+m+1\right)}\right)=\sqrt{e}
$$

likewise for

$$
\prod_{n=2}^{\infty} \frac{n^{3}-1}{n^{3}+1}=\pi \operatorname{csch} \pi
$$

we get

$$
\prod_{n=2}^{\infty} \exp \left(\frac{4 n}{n^{4}-1}\right)=\exp \left(-3 / 2+2 \gamma+\psi_{0}(2-i)+\psi_{0}(2+i)\right)
$$

we can take the product

$$
\prod_{k=1}^{\infty} \frac{\left(1+k^{-1}\right)^{2}}{\left(1+2 k^{-1}\right)}=2
$$

and find

$$
\prod_{k=1}^{\infty} \exp \left(\frac{-2}{k(k+1)(k+2)}\right)=\frac{1}{\sqrt{e}}
$$

in the same line of thought we have

$$
\begin{gathered}
\prod_{n=2}^{\infty} \exp \left(\frac{8 n^{3}}{n^{8}-1}\right)=A \\
A=\exp \left(\sum_{ \pm}-\frac{3}{2}+2 \gamma-\psi_{0}(2 \pm i)+\psi_{0}\left(2 \pm(-1)^{1 / 4}\right)+\psi_{0}\left(2 \pm(-1)^{3 / 4}\right)\right)
\end{gathered}
$$


it seems that

$$
Q_{k}=\prod_{n=2}^{\infty} \exp \left(\frac{(2 k) n^{k-1}}{n^{2 k}-1}\right)=\exp \left(\frac{3}{2}+\sum_{ \pm} \sum_{l=1}^{k-1} \psi_{0}\left(2 \pm(-1)^{l / k}\right)\right)
$$

\begin{tabular}{|l|l|l|l|}
\hline \multicolumn{4}{|c|}{ List of Products } \\
\hline$\prod_{n=2}^{\infty} \frac{n^{3}-1}{n^{3}+1}$ & $\frac{2}{3}$ & $\prod_{n=2}^{\infty} \exp \left(\frac{6 n^{2}}{n^{6}-1}\right)$ & $\sqrt{e}$ \\
$\prod_{n=1}^{\infty}\left(1+\frac{1}{n}\right)^{n} e^{1 /(2 n)-1}$ & $\frac{e^{1+\gamma / 2}}{\sqrt{2 \pi}}$ & $\prod_{n=1}^{\infty}$ & $e^{1-\gamma-\pi^{2} / 12}$ \\
\hline
\end{tabular}

